\title{
Protocolo de atenção domiciliar em enfermagem e a substitutividade
}

\author{
Rita Batistas Santos, D.Sc.*
}

Universidade Federal do Rio de Janeiro, Escola de Enfermagem Anna Nery, Departamento de Enfermagem Médico-Cirúrgica, Laboratório Interdisciplinar de Autocuidado Domiciliar, Hospital Universitário Clementino Fraga Filho, Programa de Assistência Domiciliar Interdisciplinar

\section{Resumo}

O protocolo de atenção domiciliar interdisciplinar (PADI) consiste na tabela de avaliação e manutenção das Atividades de Vida Diárias, admissão, plano terapêutico, evolução e alta domiciliar elaborado a partir do Índex de Katz, das teorias de Wanda Horta e Dorothea Orem em consonância com a legislação sobre atenção domiciliar contidas na Resolução da Diretoria Colegiada no 11 da Anvisa e da Portaria 2.529 que institui os serviços de internação domiciliar no SUS. O objetivo deste artigo é discutir a contribuição da enfermagem na construção de um protocolo de atenção domiciliar interdisciplinar. A metodologia consiste em uma análise comparativa entre as teorias de Wanda Horta e de Dorothea Orem, levantados por busca no site da BVS com a palavra-chave teorias de enfermagem nas bases de dados Lilacs e BDenf no mês de setembro de 2006, e o Index de Katz com análise interpretativa dos conceitos. Os resultados apontam que o PADI elaborado pela enfermagem orienta a assistência interdisciplinar clínico-terapêutica e psicossocial ao usuário no seu domicílio. Conclui-se que o PADI configura-se numa proposta de substitutividade, resignificando o modelo de atenção à saúde, renovando o modo hegemônico do cuidar ou desinstitucionalizando-o, criando práticas e transitando para novos campos de produção do cuidado na perspectiva da enfermagem.

Palavras-chave: enfermagem, atenção domiciliar, protocolo.

\section{Abstract \\ Home care services protocol: nursing and the substitutive proposal}

The home care services interdisciplinary protocol consists on a table of maintenance and evaluation of activities of daily living, admission, planning, evolution and outpatient home care discharge based on Katz Index, Wanda Horta and Dorothea Orem theories in accordance with legislation on home care services followed the Collegiate Directory Resolution n. 11 of Anvisa and Decree no. 2529 which establish home care hospitalization in the Single Health System. This study has as objective to discuss nursing contribution to the development of an interdisciplinary home care services protocol. The methodology consists of a comparative analysis between the theories of Wanda Horta, Dorothea Orem, applying the research in Lilacs and BDenf database using the key-word nursing theories in September 2006, and concept analysis of Katz Index. The results point out 
that the nursing protocol has guidelines for clinical and psychosocial interdisciplinary therapy in home care services. We concluded that this protocol is characterized in a substitutive proposal, giving new meaning to the health care model, renewing the hegemonic way of health care, creating new practices that promote new fields of production focusing in nursing care.

Key-words: nursing care, home care services, protocol.

\section{Resumen \\ Protocolo de atención domiciliaria en enfermería y la sustitutibilidad}

El protocolo interdisciplinar domiciliario consiste en una tabla de evaluación y mantenimiento de las actividades de la vida diaria, admisión, plan terapéutico, evolución, el alta domiciliaria elaborado a partir del índice de Katz; las teorías de Wanda Horta y de Dorothea Orem de acuerdo con la legislación de la Resolución no. 11 de la Directoria Colegiada de Anvisa y de la Portaría no. 2.529 que instituyen los servicios de internación domiciliaria en el Sistema Único de Salud. El objetivo es discutir la contribución de enfermería en la elaboración de un protocolo interdisciplinar domiciliario. La metodología consiste en un análisis comparativo entre las teorías de Wanda Horta y de Dorothea Orem, utilizando la base de datos Lilacs y BDenf y la búsqueda por la palabra clave "teoría de enfermería", en el mes de Septiembre de 2006, y el Índice de Katz con análisis de interpretación de los conceptos. Los resultados señalan que el protocolo orienta sobre la asistencia interdisciplinar clínico terapéutica y psicosocial al usuario en su domicilio. Se concluye que el protocolo configura en una propuesta de substitutividad, resignificando el modelo de atención de la salud, renovando de una forma hegemónica el cuidar, creando nuevas prácticas que promuevan nuevos campos de producción para el cuidado de enfermería.

Palabras-clave: enfermería, atención domiciliaria, protocolo.

\section{Introdução}

Com as projeçóes demográficas de envelhecimento populacional no Brasil, cresce o campo de atuação da enfermagem na Atenção Domiciliar. Com o aumento da expectativa de vida há uma necessidade de assistir a essa população, com diferentes graus de dependência, fora de instituiçóes como asilos e hospitais. Essa abordagem extra-hospitalar traz benefícios, sobretudo quando existe uma metodologia para o cuidado de enfermagem [1].

É nesse sentido que o processo de enfermagem se traduz como uma ferramenta essencial para a qualidade de vida do cliente no domicílio, tendo em vista a necessidade de regulação de competências e construção de protocolos assistenciais para enfermagem na Atenção Básica de Saúde.

Dentro dessa perspectiva, a avaliação do cliente é fundamental para a determinaçáo de todo contexto da atenção domiciliar. Para isso elaborou-se a tabela de avaliação e manutenção das Atividades de Vida Diárias (anexo 1) elaborada a partir da escala de Atividades Diárias do Index de Katz [2]; observouse a oportunidade de inter-relacionar conceitos entre as teorias de Wanda Horta [3] e Dorothea Orem [4] com as recomendaçóes da Anvisa [5] e da Portaria No. 2529 do Ministério da Saúde [5,6] configurando-se numa Política transversal na Rede SUS.

\section{Objetivos}

Este estudo pretende discutir a contribuição do referencial teórico das teorias de enfermagem de Wanda Horta, relacionada às necessidades humanas básicas afetadas, e do déficit de autocuidado de Dorothea Orem e as atividades de vida diária do Index de Katz para a construçáo de um protocolo de atenção domiciliar para o SUS.

\section{Material e método}

O estudo foi desenvolvido por meio de análise comparativa baseada na revisão de literatura sobre as teorias de enfermagem de Wanda Horta e de Dorothea Orem, o Índex de Katz e a legislação sobre atenção domiciliar no SUS.

As informaçóes e procedimentos foram levantados por busca ativa nas unidades de internação, respostas a pedidos de parecer, além de consulta a procedimentos de serviços de atenção domiciliar da rede pública e privada. Os itens de avaliação foram traduzidos numa pontuaçáo para determinar o tempo de permanência, prognóstico da autonomia, seguindo a classificaçáo em ordem crescente de dependência para o desempenho do autocuidado.

As variáveis: identificação do paciente, perfil sócio-econômico, medicamentos em uso, avaliação de desempenho e manutenção de atividades de vida 
diária do Índex de Katz foram classificadas como critérios de inclusão e exclusão para definir o perfil da clientela e as possibilidades de intervençáo à luz da revisão de literatura.

Foi realizada uma busca no site da BVS com as palavras-chave: teorias de enfermagem nas bases de dados Lilacs e BDenf no mês de setembro de 2006.

Após a leitura dos textos, foi realizada uma análise interpretativa do material coletado para discutir os conceitos afins contidos no PADI.

\section{Resultados e discussão}

A elaboração do Protocolo de Atenção Domiciliar Interdisciplinar (PADI) enfocou o levantamento de itens de avaliação voltados para a promoção da autonomia dos usuários para o autocuidado.

A RDC No.11 define como Plano de Atenção Domiciliar - PAD, o documento que contempla um conjunto de medidas que orienta a atuaçáo de todos os profissionais envolvidos de maneira direta e ou indireta na assistência a cada paciente em seu domicílio desde sua admissão até a alta, indicando a avaliação do tempo de permanência como o período compreendido entre a data de admissão e a data de alta ou óbito do paciente.

Observando a recomendação da RDC No. 11, partiu-se da admissão domiciliar (tabela de avaliação de desempenho e manutenção de atividades de vida diária - anexo 1 e folha de admissão domiciliar); plano terapêutico domiciliar; folha de evoluçáo domiciliar e plano de alta domiciliar.

A aplicação de escalas com pontuação para determinar o tempo de permanência, prognóstico do retorno as Atividades de Vida Diária (AVD'S), seguiu a classificação em ordem crescente de dependência para o desempenho do autocuidado.

A avaliação de desempenho e manutenção de atividades de vida segue a classificação em escores: valores de 0 a 15 pontos para os pacientes elegíveis para a Modalidade de Atendimento Domiciliar; de 16 a 25 pontos para os usuários elegíveis para a Modalidade de Internação Domiciliar; e acima de 25 pontos para os pacientes elegíveis para a Internação Hospitalar. Essa avaliação ajuda, ainda, a vislumbrar quanto tempo vai durar e como planejar o atendimento, classificando os usuários quanto a modalidade de Assistência Domiciliar, Internaçáo Domiciliar, ou de Internação Hospitalar e quanto tempo e freqüência de Atendimento Domiciliar
Terapêutico (ADT). Dessa forma, determinam-se parâmetros para avaliação de critérios de inclusão, permanência e manutenção de usuários bem como classificação do tipo de atenção domiciliar.

As áreas de avaliação das AVD'S listadas são Cuidador Apto, Nível de Consciência, Órteses, Curativos Ferida Operatória, Curativos de Úlceras, Suporte Ventilatório, Suporte Nutricional, Sistema Urinário, Sistema Fecal, Sistema Terapêutico Oral, Sistema Terapêutico Parenteral IM e SC, Suporte Terapêutico Venoso, Banho, Higiene Pessoal, Transferência e Mobilidade.

Diante da abordagem de Horta de um processo sistematizado de cuidar, podem-se relacionar as etapas de ambas as abordagens. Sob a perspectiva de Orem o processo se inicia com a determinação das necessidades de cuidado do indivíduo, seguida de estabelecimento de objetivos de saúde a serem alcançados por meio de um planejamento assistencial e por fim o início, a conduçáo e o controle das açôes de enfermagem, buscando compensar açôes do autocuidado e superar limitaçóes e preservar habilidades presentes de autocuidado [5].

O modelo proposto por Horta em sua etapa inicial de histórico de enfermagem propóe o levantamento de dados por meio de um roteiro sistematizado para subsidiar a etapa seguinte do diagnóstico de enfermagem, no qual as necessidades básicas afetadas do ser humano e o grau de dependência de enfermagem são determinados. Nesse sentido o grau de dependência de enfermagem deve ser classificado em total ou parcial, podendo, a enfermagem, nos casos de dependência parcial de cuidados, atuar na ajuda, orientação, supervisão e encaminhamento, quando necessário [4]. Nessa etapa o Índex de Katz se mostra útil como ferramenta para balizar o levantamento de dados, auxiliando na classificaçáo dos graus de dependência de cuidados, elegendo os clientes que atendem aos critérios para inclusão nos programas de atenção domiciliar pública no país.

A abordagem de Orem preconiza inicialmente que os diagnósticos de enfermagem devem buscar como meta a adesão do cliente ao autocuidado. Nesse sentido, apresenta uma interrelação, na medida em que a promoção do autocuidado deve trabalhar aspectos de ajuda, orientação e supervisão das atividades para capacitação do usuário.

A segunda etapa diagnóstica para Orem trabalha o planejamento dos sistemas de enfermagem, a fim de criar sistemas totalmente compensatórios, parcialmente compensatórios ou de apoio-educação, 
buscando o alcance das metas compatíveis com o diagnóstico de enfermagem [5]. Da mesma forma, Wanda Horta afirma que e terceira etapa do processo de enfermagem, chamada de plano assistencial, deve se ocupar em determinar a assistência que deve ser prestada diante do diagnóstico de enfermagem. Essa determinação leva a uma proposta de prescrição de enfermagem (quinta etapa), que trará todos os cuidados propostos de forma hierarquizada [6]. Nesse momento, tanto a quinta etapa de Horta como a última de Orem preconizam a prestaçáo da assistência prescrita; porém o modelo de Orem avança na avaliaçáo e no ajuste das atividades prescritas. Enquanto Horta utiliza mais uma etapa em seu processo, a qual atribuiu o título de evolução, para realizar a avaliação global do plano de cuidados, na qual uma estimativa busca prever a reduçáo das necessidades que se encontravam afetadas [7].

Importante ressaltar que o objetivo da assistência preconizada por Orem emerge na necessidade e preferência do indivíduo que possui liberdade e responsabilidade para alcançar o autocontrole [8]. Sendo assim o prognóstico de Orem deve levar em consideração a vontade do indivíduo de se empenhar nas atividades de autocuidado, para que as metas possam ser viáveis quanto ao alcance.

No modelo proposto por Wanda Horta o processo de enfermagem é a dinâmica das açóes sistematizadas e interrelacionadas, visando à assistência ao ser humano [3] e se divide em seis etapas: histórico de enfermagem, diagnóstico de enfermagem, plano assistencial, prescrição de enfermagem, evoluçáo e prognóstico.

Nesse processo, na etapa de histórico, a enfermeira realiza o levantamento de dados que irá subsidiar a etapa seguinte de diagnóstico, na qual é realizado um julgamento clínico identificando as necessidades humanas afetadas (fisiológicas, de segurança, de amor, de estima e de auto-realizaçáo). Cada necessidade deve ser atendida hierarquicamente através do processo de enfermagem. Seu plano assistencial compreende a etapa em que as metas e as açóes de enfermagem são planejadas para subsidiar a etapa de prescrição de enfermagem. Sua evoluçáo de enfermagem observa as alteraçóes no quadro do usuário. Seu processo é finalizado com a etapa de prognóstico onde a potencialidade de atendimento das necessidades humanas afetadas naquele usuário é estimada.

O modelo conceitual de Orem se baseia em três alicerces teóricos básicos: a teoria do autocuidado, a teoria do déficit do autocuidado e a teoria dos sistemas de enfermagem [4]. Dentro dessa perspectiva, o autocuidado e o cuidado de pessoas dependentes são comportamentos que regulam a integridade, o funcionamento e o desenvolvimento humano, relacionando açóes de autocuidado e seu desenvolvimento [8].

A teoria do déficit de autocuidado postula que a necessidade de cuidados de enfermagem existe à medida que as pessoas possuem algum tipo de limitação que as incapacite de cuidar de si mesmo ou de seus dependentes.

Orem na teoria dos sistemas de enfermagem classificou-os em: sistema totalmente compensatório, onde o ser humano é incapaz de promover seu autocuidado; o sistema parcialmente compensatório em que o enfermeiro e o cliente promovem cuidados relativos à manipulação ou à locomoção; e o sistema de apoio-educação - o cliente consegue executar medidas de autocuidado terapêutico, mas necessita de auxílio [8].

O Índex de Katz proposto por Sidney Katz é um modelo de avaliaçáo funcional da capacidade de se autocuidar e atender as atividades de vida diária (AVD). Destacam-se dentro do universo das AVD`s básicas as capacidades de: alimentar-se, vestir-se, transferir-se, banhar-se, ter continência e usar o banheiro [10]. Essas atividades situam-se entre as seis áreas básicas que se incluem na avaliação do autocuidado. De acordo com o Índex, a pessoa terá sua capacidade diante dessas atividades classificada como independente (zero em cada atividade) ou dependente (um ponto em cada atividade), sendo esta última atribuída mesmo aos indivíduos que têm aparentemente essa capacidade, mas que se recusam realizar uma atividade prevista [2].

Ao aplicar o Índex, podemos afirmar que quanto maior a pontuação obtida, maior é o grau de dependência.

Diante da abordagem de Horta um processo sistematizado de cuidar pode-se relacionar às etapas de ambas as abordagens. Sob a perspectiva de Orem, o processo se inicia com a determinação das necessidades de cuidado do indivíduo, seguida de estabelecimento de objetivos de saúde a serem alcançados por meio de um planejamento assistencial e por fim o início, a condução e o controle das açôes de enfermagem, buscando compensar açôes do autocuidado e superar limitaçóes e preservar habilidades presentes de autocuidado [4].

A respeito dos tipos de Serviços de Atenção Domiciliar - SAD prestados por instituição pú- 
blica ou privada, responsável pelo gerenciamento e operacionalização de assistência e/ou internação domiciliar, a RDC No.11 subdivide-se em: Assistência Domiciliar (conjunto de atividades de caráter ambulatorial, programadas e continuadas desenvolvidas em domicílio) e Internaçáo Domiciliar [5]. Recomenda que o $S A D$ possua um regimento interno que defina o tipo de atenção domiciliar prestada e as diretrizes básicas que norteiam seu funcionamento e, além disso, elabore um manual e normas técnicas de procedimentos para a atenção domiciliar, de acordo com a especificidade da assistência a ser prestada [5]. Neste sentido, a construção do protocolo de atenção domiciliar interdisciplinar pela enfermagem contribui tanto para a organizaçáo do processo de trabalho interdisciplinar quanto para a indicação do tipo de atenção domiciliar a ser adotada pelo profissional de saúde que acompanha o paciente.

O Formulário de Encaminhamento do PADI é um relatório detalhado sobre as condiçóes de saúde e doença do paciente contendo histórico, prescriçóes, exames e intercorrências, informaçôes decisivas para a elaboração do Plano Terapêutico de Atenção Domiciliar - PAD.

A Evolução Domiciliar é a parte do PAD que deve contemplar: a prescriçáo da assistência clínico-terapêutica e psicossocial para o paciente, requisitos de infra-estrutura do domicílio do paciente, necessidade de recursos humanos, materiais, medicamentos, equipamentos, retaguarda de serviços de saúde, cronograma de atividades dos profissionais e logística de atendimento. Ele fornece a noção do tempo estimado de permanência do usuário no atendimento considerando a evolução clínica, superação de déficits, autonomia para o autocuidado ou independência de cuidados técnicos e de medicamentos, equipamentos e materiais que necessitem de manuseio continuado de profissionais. A periodicidade dos relatórios de evolução e acompanhamento estará de acordo com a evolução e acompanhamento do paciente e a gravidade do caso contendo data, assinatura do profissional de saúde que acompanha o paciente e do responsável técnico do SAD.

O PADI deve ser preenchido com letra legível e pode ser assinado por todos os profissionais envolvidos diretamente na assistência. Após a alta ou óbito do paciente, o prontuário deve ser arquivado, conforme legislação vigente, com o fornecimento de cópia integral do prontuário quando solicitado pelo usuário ou pelos responsáveis legais.
Segundo a Portaria no. 2.529, de 19 de outubro de 2006, a atenção domiciliar integra duas modalidades específicas, a assistência domiciliar e a internação domiciliar. A elaboração do Protocolo de Atenção Domiciliar Interdisciplinar (PADI) serve a prestação da assistência ou internação domiciliar.

A Portaria 2529 considera que a assistência domiciliar no âmbito do SUS é desenvolvida por profissionais da atenção básica e/ou da atenção especializada. Os Programas de Saúde de Família e Agentes Comunitários de Saúde não constituem, a princípio, programas de atenção domiciliar terapêutica. Podem vir a sê-lo, na dependência dos recursos alocados e de sua articulação com outros níveis de assistência.

A abordagem integral ao usuário/família significa considerá-los em seu contexto sócio-econômico e cultural, dentro de uma visão ética compromissada com o respeito e a individualidade. Assistir com integralidade inclui, entre outras questóes, conceber o homem como sujeito social capaz de traçar projetos próprios, intervindo dinamicamente nos rumos do cotidiano.

A assistência no domicílio deve conceber a singularidade da família em seu espaço social e doméstico, respeitando o movimento e a complexidade das relaçóes familiares. Ao profissional de saúde que se insere na dinâmica da vida familiar cabe uma atitude de respeito e valorização das características peculiares daquele convívio humano. Assim, a abordagem integral deve ser sempre considerada por envolver múltiplos fatores no processo saúde-doença da família, influenciando as formas de cuidar.

Neste sentido, procura a construção de ambientes mais saudáveis para a pessoa em tratamento que envolve, além do conhecimento profissional, o reconhecimento das potencialidades terapêuticas presentes nas relaçôes familiares. Os conflitos, as interações e desagregações fazem parte do universo simbólico e particular da família, intervindo diretamente na saúde de seus membros. Assistir no domicílio é cuidar da saúde da família com integralidade e dinamicidade, reconstruindo relaçóes e significados.

Por isso, a Portaria 2529 institui a Internação Domiciliar no âmbito do Sistema Único de Saúde SUS, definindo-a como um conjunto de atividades prestadas no domicílio a pessoas clinicamente estáveis que exijam intensidade de cuidados acima das modalidades ambulatoriais, mas que possam ser mantidas em casa, por equipe exclusiva para este fim.

A classificação proposta no PADI esclarece os critérios de inclusão que possibilitem determinar 
o tipo ou modalidade de atenção domiciliar em virtude da lacuna entre as definiçóes de Assistência Domiciliar e Internação domiciliar no que se refere à definição da inserção dos PSF's neste contexto e vislumbra um horizonte neste sentido.

Obedecendo a Portaria 2.529, o PADI estabelece como prioridade de eleição os seguintes grupos populacionais: idosos; portadores de doenças crônico-degenerativas agudizadas; portadores de patologias que necessitem de cuidados paliativos; e portadores de incapacidade funcional, provisória ou permanente. Entretanto, abre espaço para outras prioridades, além das definidas, privilegiando aquelas que poderão ser estabelecidas localmente.

O PADI indica como critérios de exclusão de usuários, dada a complexidade do modelo proposto, os usuários com perfil para internação hospitalar, entretanto estimula que outros critérios de exclusão podem ser definidos pelos protocolos locais de acordo com a infra-estrutura de equipamento e recursos humanos disponíveis.

\section{Conclusão}

Podemos concluir que há relação entre os processos de enfermagem de Wanda Horta e Dorothea Orem, em etapas distintas, no que se refere à aplicação simultânea de ambos na prestação da atenção domiciliar, onde a adesão do usuário ao autocuidado é uma meta a ser alcançada, na medida em que a abordagem para a sua promoção trabalha aspectos de ajuda, orientação e supervisão das atividades para independência/autonomia do usuário, família e comunidade. Com isso conclui-se que a interrelaçáo entre as teorias pode auxiliar no incentivo ao autocuidado através da abordagem das necessidades humanas básicas afetadas e das AVD's.

O PADI orienta a Equipe Multidisciplinar de Atenção Domiciliar - EMAD cujos profissionais têm a função de prestar assistência clínico-terapêutica e psicossocial ao usuário em seu domicílio.

Por meio desta avaliação de enfermagem é possível contribuir para a desospitalização de eventos desnecessários, estimulando processos de "alta precoce", evitando intercorrências hospitalares em pacientes crônicos, com histórico de reinternaçóes, humanizando a atenção de enfermagem hospitalar e em alguns casos garantindo uma morte digna.

É importante que a enfermeira possa trabalhar aspectos relevantes das teorias buscando complementá-las, a fim de que os cuidados prestados possam ser subsidiados por modelos teóricos próprios da profissão que podem ser interrelacionados; buscando uma assistência à comunidade pautada em modelos que atendam às demandas do usuário não só no cenário hospitalar, mas também no que se refere à promoção da saúde na atenção domiciliar.

O PADI configura-se numa interface entre os modelos das teorias de enfermagem e as AVD'S, criando novos campos de prática de produção do cuidado transitando para novos campos para a produção do cuidado em saúde a partir da perspectiva da enfermagem.

A Sistematização da Atenção Domiciliar Interdisciplinar pela enfermagem contribui para produção e gerenciamento de serviços de atençáo domiciliar, segundo as recomendaçôes da RDC 11 da Anvisa e da Portaria 2.529 do Ministério da Saúde.

\section{Agradecimentos}

Este trabalho é parte de projeto de avaliação de um protocolo de atenção domiciliar interdisciplinar pela enfermagem recomendado e aprovado pela Diretoria Executiva do CNPq através do edital universal 2006.

\section{Referências}

1. Duarte YAO, Diogo MJDE. Atendimento domiciliar: um enfoque gerontológico. São Paulo: Atheneu; 2000.

2. Roach S. Introdução à Enfermagem Gerontológica. Rio de Janeiro: Guanabara Koogan; 2003.

3. Horta WA. Processo de Enfermagem. São Paulo: EPU; 1979.

4. Foster PC, Janssens NP. In: George JB, et al. Teorias de Enfermagem. Porto Alegre: Artes Médicas; 1993. p. 90-107.

5. Agência Nacional de Vigilância Sanitária. Resolução da Diretoria Colegiada, RDC no 11, de 26 de janeiro de 2006. Brasília: D.O.U. de 30 de Janeiro de 2006.

6. Ministério da Saúde. Lei no 10.424 , de 15 de abril de 2002. Brasília: MS; 2002.

7. Merhy EE. Feuerwerker LM. Atenção domiciliar: medicalização e substituvidade [online]. [citado em 2007 Mai 2]. Disponível em URL: http://www.hucff.ufrj.br/ micropolitica/pesquisas/atencaodomiciliar/textos.php

8. Dupas G, Pinto IC, Mendes MD, Benedini Z. Reflexão e síntese acerca do modelo do auto-cuidado de Orem. Acta Paul Enfermagem 1994;7(1):19-26.

9. Diogo MJDE. O papel da enfermeira na reabilitação do idoso. Rev Latinoam Enfermagem 2000;8(1):75-81.

10. Schier J, Gonçalves LHT. Grupo aqui e agora - uma tecnologia leve de ação socioeducativa de enfermagem. Texto e Contexto Enfermagem 2005;14(2):271-279. 


\section{Anexo 01}

Avaliação de desempenho e manutenção de atividades de vida (Estimativa de tipo, prognóstico de alta e permanência do usuário)

A partir da pontuação atribuída a cada item determinar o tempo de permanência, prognóstico do retorno as AVD'S, seguindo a classificação abaixo.

A - Escore Inferior a 15 pontos - Elegível para a Modalidade de Atendimento Domiciliar:

A1 - até 05 pontos - suporte terapêutico e social - independente para as AVD'S - permanência de dias em atendimento domiciliar, com atendimentos semanais;

A2 - 06 a 10 pontos - parcialmente independente - permanência de dias em atendimento domiciliar, com atendimentos semanais;

A3 - 11 a 15 pontos - parcialmente dependente - permanência de dias em atendimento domiciliar, com atendimentos semanais.

B - Escore de 16 a 25 pontos - Elegível para a Modalidade de Internação Domiciliar:

B1 - 16 a 20 pontos - Elegível para a Modalidade de Internação Domiciliar - Baixa Complexidade, parcialmente dependente - permanência de dias em atendimento domiciliar, com atendimentos semanais.

B2 - 21 a 25 pontos - Elegível para a Modalidade de Internação Domiciliar - Média Complexidade, totalmente dependente - permanência de dias em atendimento domiciliar, com atendimentos semanais.

C - Acima de 25 pontos - Elegível para a Internação Hospitalar.

\begin{tabular}{|c|c|c|}
\hline Áreas de avaliação & Itens de avaliação & Pontuação \\
\hline \multirow[t]{2}{*}{ Cuidador apto } & 0. Sim & \\
\hline & 1. Não & \\
\hline \multirow[t]{5}{*}{ Nível de Consciência } & 0. Alerta/Orientado & \\
\hline & 1. Alerta/Desorientado & \\
\hline & 2. Torporoso & \\
\hline & 3. Coma transitório & \\
\hline & 4. Coma persistente & \\
\hline \multirow[t]{5}{*}{ Órteses } & 0. Deambula livremente & \\
\hline & 1. Usa muletas c/ ajuda & \\
\hline & 1. Cadeira de Rodas c/ ajuda & \\
\hline & 1. Uso de Andador c/ ajuda & \\
\hline & 2. Acamado & \\
\hline \multirow[t]{5}{*}{ Curativos ferida operatória } & 0. Não necessita & \\
\hline & 1. Pequeno & \\
\hline & 2. Médio & \\
\hline & 3. Grande & \\
\hline & Infectado - acrescentar +1 & \\
\hline \multirow[t]{5}{*}{ Curativos de úlceras } & 0. Não necessita & \\
\hline & 1. Grau 1 & \\
\hline & 2. Grau 2 & \\
\hline & 3. Grau 3 & \\
\hline & 4. Grau 4 & \\
\hline \multirow[t]{8}{*}{ Suporte ventilatório } & 0. Ar ambiente & \\
\hline & 1. Cateter & \\
\hline & 2. Nebulização & \\
\hline & 2. Traqueostomia sem aspiração & \\
\hline & 3. Macronebulização Intermitente & \\
\hline & 4. Macronebulização contínua & \\
\hline & 5. Traqueostomia com aspiração & \\
\hline & 5. Prótese Ventilatória & \\
\hline
\end{tabular}




\begin{tabular}{|c|c|c|}
\hline Áreas de avaliação & Itens de avaliação & Pontuação \\
\hline \multirow[t]{7}{*}{ Suporte nutricional } & 0. Não necessita & \\
\hline & 1. Suplemento Oral & \\
\hline & 1. Alimenta-se com Assistência & \\
\hline & 2. Gastrostomia & \\
\hline & 3. Sonda Nasoenteral & \\
\hline & 3. Jejuno-ileostomia & \\
\hline & 5. Parenteral & \\
\hline \multirow[t]{4}{*}{ Sistema urinário } & 0. Controla Esfíncteres & \\
\hline & 1. Dispositivo Urinário Externo & \\
\hline & 2. Sonda Vesical & \\
\hline & 3. Sonda Supra Púbica (Cistostomia) provisória & \\
\hline \multirow[t]{3}{*}{ Sistema Fecal } & 0. Controla Esfíncteres & \\
\hline & 1. Uso de Fralda Geriátrica & \\
\hline & 2. Colostomia & \\
\hline \multirow[t]{4}{*}{ Sistema Terapêutico Oral } & 0. Não necessita/Não Necessita orientação & \\
\hline & 1. Sintomáticos & \\
\hline & 1. Uso regular & \\
\hline & Necessita Orientação -+1 & \\
\hline \multirow{3}{*}{$\begin{array}{l}\text { Sistema Terapêutico Parenteral IM E } \\
\text { SC }\end{array}$} & O. Não necessita & \\
\hline & 1. Uso intermitente & \\
\hline & 2. Uso contínuo & \\
\hline \multirow[t]{5}{*}{ Suporte Terapêutico Venoso } & 0. Não necessita & \\
\hline & 1. Acesso Venoso Periférico & \\
\hline & 3. Acesso Venoso Profundo & \\
\hline & 3. Cateter Implantado & \\
\hline & Freqüência de uso $1 x-+1$ & \\
\hline \multirow[t]{2}{*}{ Banho } & 0. Sem ajuda & \\
\hline & 1. Não toma banho sozinho & \\
\hline \multirow[t]{3}{*}{ Vestuário } & $\begin{array}{l}\text { 0. Escolhe a roupa e veste-se completamente sem } \\
\text { assistência. }\end{array}$ & \\
\hline & $\begin{array}{l}\text { 1. Escolhe a roupa e veste-se sem assistência, exceto } \\
\text { para amarrar os sapatos. }\end{array}$ & \\
\hline & $\begin{array}{l}\text { 1. Recebe assistência para escolher a roupa ou para } \\
\text { vestir-se ou não fica vestido, total ou parcialmente. }\end{array}$ & \\
\hline \multirow[t]{3}{*}{ Higiene Pessoal } & $\begin{array}{l}\text { 0. Vai ao banheiro, se limpa e arruma as roupas sem } \\
\text { assistência. }\end{array}$ & \\
\hline & $\begin{array}{l}\text { 1. Recebe Assistência para ir ao banheiro, para } \\
\text { limpar-se ou para arrumar as roupas após elimina- } \\
\text { ção ou no uso de fraldas, papagaio ou comadre. }\end{array}$ & \\
\hline & 2. Não vai ao banheiro para eliminações fisiológicas. & \\
\hline \multirow[t]{3}{*}{ Transferência/Mobilidade } & 0. Mobiliza-se sem ajuda & \\
\hline & $\begin{array}{l}\text { 1. Move-se na cama ou para fora dela, bem como } \\
\text { senta-se ou sai da cadeira sem assistência. }\end{array}$ & \\
\hline & $\begin{array}{l}\text { 2. Move-se na cama ou para fora dela com assistên- } \\
\text { cia. }\end{array}$ & \\
\hline \multicolumn{2}{|l|}{ TOTAL DE PONTOS } & \\
\hline
\end{tabular}

